- Tout progrès en ce domaine suppose une multi-disciplinarité associant métallurgistes, céramistes, polyméristes, biologistes, stomatologues, chirurgiens... C'est ainsi que de nouveaux biomatériaux seront mis au point, pour atteindre des durées de vie supérieures, diminuer les risques postopératoires, améliorer le confort des patients et pouvoir reconstituer des organes entiers. Un effort important doit être réalisé dans l'ensemble de ce domaine.

\title{
10 Emballage
}

\subsection{Enjeux}

L'emballage constitue un marché important. Parmi les différents matériaux utilisés en France (chiffre d'affaires : 17,3 milliards d'euros en 2007), la part du métal représente $13 \%{ }^{8}$, parmi lesquels les boîtes de conserve $32 \%$, les boîtes boisson $15 \%$ et le bouchage $13 \%$ constituent l'essentiel ${ }^{9}$.

L'environnement et la sécurité pour les emballages alimentaires s'imposent de plus en plus. On doit donc se préoccuper :

- du recyclage des emballages, point favorable pour les métaux;

- de leur contenu énergétique, donc en particulier de la masse ;

- de la protection du contenu, tout en se pliant aux exigences de l'esthétique et du marketing (annexe 18).

\subsection{Métallurgie, protection et recyclage}

L'acier et les alliages d'aluminium se partagent l'essentiel du marché de l'emballage métallique, la recherche se concentrant sur le secteur de l'agroalimentaire, où l'emballage rigide compte pour $\approx 70 \%$. Enjeux et applications sont à peu près les mêmes pour ces deux matériaux qui sont donc concurrents frontaux sur ce marché très disputé.

L'acier doit être recouvert d'un revêtement pour éviter le contact avec les aliments. La solution traditionnelle consiste à le recouvrir d'un film fin d'étain : $c^{\prime}$ est le « fer blanc ». Plus récemment a été développée la famille des «tin-free steels (TFS) où l'on utilise notamment le titane, les boîtes étant en outre recouvertes d'un vernis. Le métal de base est un acier ferritique durci par du carbone.

\footnotetext{
${ }^{8}$ Plastique : $35 \%$; papier-carton : $32 \%$; verre : $11 \%$; bois : $9 \%$.

${ }^{9}$ Source : SESSI (INSE), enquête annuelle de branche, 2007.
} 
On s'oriente actuellement vers des techniques de refroidissements rapides. Pour l'aluminium, la couche d'alumine revêtue $d^{\prime}$ un vernis sert de protection et les alliages pour emballage rigide sont obtenus par corroyage au laminage d'alliages 3XXX (Al-Mn) et 5XXX (Al-Mg-Mn). Pour améliorer encore la protection des aliments, les recherches se poursuivent sur des revêtements par dépôt sous vide ou sur de nouveaux polymères.

Les capacités de recyclage sont bonnes pour les deux matériaux. L'acier est trié par magnétisme, l'étain du revêtement étant vaporisé lors de la refonte en four électrique et se retrouvant dans les poussières, objet de recherches poussées. Les boîtes en aluminium sont triées par courants de Foucault dans les centres de tri sélectif. Elles sont ensuite fondues en four à couverture de bain de sels fondus (four rotatif basculant), et recyclées dans la même filière. Ce procédé fait l'objet de travaux de R\&D visant à maximiser le rendement et l'efficacité énergétique.

\subsection{Résistance mécanique et mise en forme}

Afin de diminuer les coûts et le contenu énergétique en $\mathrm{CO}_{2}$, une course à l'amincissement est engagée. Aujourd'hui des épaisseurs de 80-100 $\mu \mathrm{m}$ sont courantes. La propreté en inclusions est cruciale pour la mise en forme par étirage du corps des boîtes boisson à des épaisseurs finales aussi faibles. De plus, la fabrication à haute cadence des boîtes doit être assurée sans grippage de la bande sur les bagues d'étirage. Dans le cas de l'alliage d'aluminium 3104-H19, un mécanisme d'autonettoyage de la bague, par les composés de phases intermétalliques au manganèse dispersés, le permet.

Les boîtes devant résister à la pression intérieure, notamment durant la stérilisation, ainsi qu'aux manipulations lors du stockage, il faut trouver des alliages plus résistants mais conservant la même facilité de mise en forme. Pour l'acier comme pour l'aluminium, le durcissement est obtenu en optimisant éléments $d^{\prime}$ addition et écrouissage, tout en conservant une bonne ductilité. Difficulté particulière : l'écrouissage induit une orientation préférentielle (texture) des grains, $d^{\prime}$ 'où une déformation inhomogène du métal (formation de " cornes " d'emboutissage). Le compromis entre un taux de cornes acceptable et un durcissement suffisant est en continuelle amélioration. Sa maîtrise nécessite des modèles de comportement très précis.

L'aluminium est aussi utilisé dans divers emballages, semi-rigides (barquettes, tubes, opercules) ou souples, tels que les complexes multicouches pour briques associant feuille mince, jusqu'à $6 \mu \mathrm{m}$, en aluminium faiblement allié, polymère et carton. On doit alors optimiser les performances des fevilles en jouant sur les procédés (coulée continue ou semi-continue, laminage, traitements thermiques) 
et la microstructure (distribution des particules intermétalliques, structure granulaire fine).

\subsection{Position de la France}

Le marché des bandes pour emballages rigides est essentiellement européen et concentré, avec de grands clients dont les lignes de remplissage sont proches des centres de productions des boissons ou aliments.

La France dispose de deux centres de recherche industriels de très bon niveau (déjà cités) à Voreppe pour les alliages d'aluminium et à Maizières-lèsMetz pour les aciers. Des équipes universitaires travaillent sur la maîtrise des séquences de précipitation dans l'acier, sur les textures, la mise en forme et l'élaboration, avec une compétence particulière en tribologie (Lyon et SophiaAntipolis). Une forte présence d'universités européennes telles que celle d'Aix-la Chapelle $\left(\mathrm{RWTH}^{10}\right)$ sur ces sujets doit être soulignée.

Au total, le marché de l'emballage métallique pour l'agro-alimentaire (conserve, boisson) est important pour la Métallurgie française qui est leader dans la fourniture de laminés acier (ArcelorMittal) et aluminium (Alcan Tôles de Spécialités).

- En termes de recommandations s'impose ici le maintien d'une R\&D de haut niveau scientifique et technique. Elle concerne tous les métiers (recyclage, élaboration, coulée, laminage, Métallurgie des produits, formage), en une approche globale cumulant les améliorations incrémentales à chaque stade de la fabrication des demi-produits et des emballages, et associant étroitement client et fournisseur. Elle vise à des améliorations continues des performances (réduction des épaisseurs) et des procédés (fonderie, laminage, formage). Les compétences des laboratoires français sont à préserver :

- en métallurgie physique de la mise en forme (texture-anisotropie, endommagement);

- en modélisation numérique des procédés de mise en forme (laminage, parachèvement), avec les mécaniciens;

- selon des approches multi-physiques, sur les procédés de recyclage (haute température) et sur la lubrification et la tribologie en laminage.

\footnotetext{
${ }^{10}$ RWTH : Rheinisch-Westfãlische Technische Hochschule - Université technique de RhénanieWestphalie.
} 